appropriateness of follow up timing between BO experts (those performing $>50$ gastroscopies for surveillance or therapy per year) and non-experts, was further analysed by referrer on the order request. There was a significantly better adherence to follow up timing when the referrer was an expert $(p=0.011)$.

Conclusions High quality endoscopic surveillance relies both on high quality endoscopy as well as appropriate decision making around the timing of surveillance. Adherence to guidelines for the timing of follow up endoscopy is poor particularly in short segment Barrett's and particularly by non-expert Barrett's endoscopists. This argues for better vetting by experts to decide the timing of follow up endoscopies.

\section{PTH-70 ASSESSMENT OF CONCORDANCE BETWEEN OESOPHAGEAL HRM + PHMETRY, AND BARIUM SWALLOWS}

${ }^{1}$ Harriett Warrior*, ' ${ }^{1}$ Caroline Race, ${ }^{1}$ Kate Mason, ${ }^{1,2}$ Ragu Vinayagam, ${ }^{1}$ Stuart Riley. ${ }^{1}$ Sheffield Teaching Hospitals, Sheffield, UK; ${ }^{2}$ Pennine Acute Hospitals NHS Trust, UK

\subsection{6/gutjpl-2021-BSG.249}

Introduction To determine the sensitivity and specificity of barium swallow in reference to the gold standard HRM +24 hour $\mathrm{pH}$ studies to identify abnormalities in peristalsis, lower oesophageal sphincter (LOS) relaxation and detection of gastro-oesophageal reflux (GOR) in symptomatic patients. Concordance of oesophageal peristalsis and LOS relaxation between conventional oesophageal manometry and barium swallow has been well documented in synchronous and asynchronous studies. However, research comparing barium swallow and oesophageal HRM is limited and there is sparse data evaluating the ability of barium studies to detect GOR in comparison to 24 hour $\mathrm{pH}$ studies.

Methods 88 patients with GOR and/or dysphagia uncontrolled by medication, who have undergone HRM +/- 24 hour $\mathrm{pH}$ studies and a barium swallow within 12 months of each other, were identified. Consecutive patients were selected upon referral to Northern General Hospital between February 2018 and June 2019, with the exception of those without a gastroscopy to rule out structural abnormalities and those who have a history of upper gastrointestinal surgery. Of the 88 patients initially included in the study, data for 48 subjects was available to be blindly reviewed. The barium swallow images were then blindly reviewed by a consultant radiologist and oesophageal manometry +24 hour $\mathrm{pH}$ results were reviewed by a clinical scientist to determine if inter-observer variability is a confounding variable.

Results 88 patients met inclusion criteria; of which we had available data on peristalsis for 88 , data on LOS relaxation for 85 and reflux for 72 . Referenced to HRM, barium swallow indicated $40 \%$ sensitivity and $53 \%$ specificity for identifying abnormal peristalsis, $61 \%$ sensitivity and $98 \%$ specificity for detecting abnormal LOS relaxation and 51\% sensitivity and $72 \%$ specificity for detecting reflux. When assessing concordance between the reviewed manometry and barium swallows, there was little change within the peristalsis and reflux parameters, with the greatest change observed for LOS relaxation.

Conclusion In this tertiary centre, concordance between HRM and barium swallow is poor, and specifically, barium swallow is lacking in ability to identify abnormalities in oesophageal peristalsis and the reviewed data suggests that inter-observer variation is a factor causing discrepancies in the assessment of LOS relaxation. Further, in those patients whose results were changed when reviewed, these changes are unlikely to have affected their clinical management.

\section{PTH-71 EXPLORING THE ROLE OF DEDICATED SERVICES FOR BARRETT'S OESOPHAGUS CARE IN UK NHS HOSPITALS}

${ }^{1,2}$ Elizabeth Ratcliffe*, ${ }^{2,3}$ Yong Liew, ${ }^{2,4}$ Jen Kuan, ${ }^{2,5}$ Yeson Kim, ${ }^{2,6}$ Maja Kopczynska, 2,6 James Britton, ${ }^{2,6}$ John McLaughlin, ${ }^{2,6}$ Shaheen Hamdy, ${ }^{2,6}$ Yeng Ang. 'Wrightington Wigan and Leigh NHS Trust; ${ }^{2}$ University of Manchester; ${ }^{3}$ East Lancashire Hospitals NHS Trust; ${ }^{4}$ Lancashire Teaching Hospitals NHS Trust; ${ }^{5}$ Borders General Hospital; ${ }^{6}$ Northern Care Alliance NHS Trust

\subsection{6/gutjnl-2021-BSG.250}

Introduction Barrett's oesophagus (BO) services in the UK are run with different models. Though it is not currently advised in guidelines, studies suggest a benefit from dedicated services showing better adherence to surveillance protocols and concomitant dysplasia detection [1,2]. As the extent of BO dedicated services in the UK has not been reported in the literature, we aimed to gain insight into the extent of this practice in NHS trusts.

Methods Adult NHS endoscopy units in the UK known to the Joint Advisory Committee (excluding paediatric units) were contacted via phone or email and asked to complete a 6-question survey about the $\mathrm{BO}$ endoscopy service provided in their unit.

Results In total, 249 departments ( 95\%) were contacted with a response rate of $62.2 \%(155 / 249)$. Of these $140 / 155$ (90.3\%) performed BO surveillance in their unit and 83/140 (59.3\%) reported having a dedicated service. When asked who was running the service, most reported a mixture of gastroenterologists, surgeons, nurses and fellows, with 22/83 (26.5\%) reporting a single clinician. Regarding specific interventions and techniques, having a dedicated BO service was significantly associated with the use of high-resolution white light (92.8\% vs $70 \% \mathrm{p}=<0.0001)$ and acetic acid $(84.3 \%$ vs $50 \%$ $\mathrm{p}<0.0001)$. There was no significant difference for virtual chromoendoscopy techniques $(\mathrm{p}=0.12)$ or dye chromoendoscopy $(p=0.12)$.

Having a dedicated endoscopy $\mathrm{BO}$ service was associated with local availability of radiofrequency ablation $(43.4 \%$ vs $18.3 \% \mathrm{p}=0.002)$, endoscopic ultrasound $(63.9 \%$ vs $30 \% \mathrm{p}$ $<0.0001)$, endoscopic mucosal resection $(60.24 \%$ vs $38.3 \%$ $\mathrm{p}=0.015)$ and endoscopic submucosal dissection $(34.9 \%$ vs $5 \% \mathrm{p}<0.0001)$. However, many trusts without these services also reported a dedicated $\mathrm{BO}$ endoscopy service suggesting practice in non-tertiary setting. There was no significant difference in provision of a dedicated $\mathrm{BO}$ service when accounting for JAG accreditation status $(\mathrm{p}=0.9)$.

Trusts which reported a dedicated endoscopy service were significantly more likely to also have a dedicated Barrett's clinic $(54.2 \%$ vs $16.7 \% \mathrm{p}<0.0001)$ and have a named lead for Barrett's within their unit $(86.8 \%$ vs $33.3 \% \mathrm{p}<0.0001)$

Conclusions Dedicated services for $\mathrm{BO}$ are provided in the NHS, and our study suggests that quality of care indicators such as use of high-resolution white light endoscopy are linked with dedicated services. Studies should be undertaken to measure the outcomes for patients with cost benefit analysis to determine the efficacy and efficiency of this service to determine best practice. 


\section{REFERENCES}

1. Britton J, Chatten K, Riley T, Keld RR, Hamdy S, McLaughlin J, et al. Dedicated service improves the accuracy of Barrett's oesophagus surveillance: a prospective comparative cohort study. Frontline Gastroenterol. 2019 Apr;10(2):128-34.

2. Ooi J, Wilson P, Walker G, Blaker P, DeMartino S, O'Donohue J, et al. Dedicated Barrett's surveillance sessions managed by trained endoscopists improve dysplasia detection rate. Endoscopy. 2017 Jun;49(6):C1.

\section{PTH-72 A SYMPTOM AND RISK FACTOR QUESTIONNAIRE ACCURATELY PREDICTS UPPER GASTROINTESTINAL CANCER}

${ }^{1}$ Kai Man Alexander Ho*, ${ }^{2}$ Avi Rosenfeld, ${ }^{1}$ Áine Hogan, ${ }^{1}$ Hazel McBain, ${ }^{1}$ Margaret Duku, ${ }^{1}$ Paul Wolfson, ${ }^{1}$ Ash Wilson, ${ }^{1}$ Study Group SPIT, ${ }^{3}$ David Graham, ${ }^{3}$ Vinay Sehgal, 1,3 Laurence Lovat. 'University College London, London, UK; ${ }^{2}$ Jerusalem College of Technology, Jerusalem, Israel; ${ }^{3}$ University College London Hospitals NHS Trust, London, UK

\subsection{6/gutjnl-2021-BSG.251}

Introduction Waiting times for endoscopy are rising rapidly following the COVID-19 pandemic. In addition, cancers may be missed as patients are placed on routine waiting lists but not monitored. Some hospitals use the Edinburgh Dysphagia Score to assess and prioritise patients for investigation. This offers a sensitivity of $98.4 \%$ and specificity of $9.3 \%$ to detect malignancy in patients presenting with dysphagia. ${ }^{4}$ However, it is not designed for detecting gastric cancer. We aimed to create a more accurate screening questionnaire as an aid to triaging referrals.

Methods Patients were recruited as part of the Saliva to Predict rIsk of disease using Transcriptomics and epigenetics (SPIT) study. Patients were recruited from 2 week-wait suspected upper gastrointestinal cancer pathway referrals at 20 hospitals in the United Kingdom. The cohort was further enriched with patients found to have oesophageal adenocarcinoma on emergency hospital admission. They completed over 200 questions about a wide variety of symptoms and risk factors. After data cleaning, 800 patients were available for evaluation. Of these, 80 had upper GI cancer. A machine learning model was developed to identify those at highest risk of having upper GI cancer using a 'cost-based' approach which maximises the chance of detecting cancer. Information gain was followed by correlated feature selection and a multivariable logistic regression curve was created with scores from 0 (cancer very unlikely) to 100 (cancer very likely). The training dataset used $80 \%$ of the data and the model was tested with the other $20 \%$.

Results 20 features were found to be important and reproducible. They included age, sex, dysphagia, odynophagia, early satiety, weight loss, duration of chest pain and regurgitation, frequency of acid taste in the mouth, a previous history of smoking, cancer or psychological disorders, current anxiety level and frequency of vegetable intake. The area under the receiver operator curve to detect cancer was 0.83 . $50 \%$ of cancers scored greater than 85 whereas $50 \%$ of normals scored less than 25. At a cut-off score of 10 , sensitivity was $98.7 \%$ with specificity $26.8 \%$ to detect cancer (figure).

Conclusions We have created a simple, reproducible risk score to identify patients at high and low risk of upper GI cancer. It performs better than previous scores but now needs testing in the real world. It might be usable to both upgrade routine patients to urgent endoscopy and remove patients at very low risk from waiting lists, thereby helping to prioritise patients with a greater clinical need and reducing the endoscopic backlog.

\section{PTH-73 BENCHMARK AUDIT OF THE MANAGEMENT OF POST- OESOPHAGECTOMY BARRETT'S}

Matthew Harborne*, Amar Mann, James Gale, Gowshan Rajeswaran, Ralph Boulton, M Adil Butt. Queen Elizabeth Hospital, Birmingham, Birmingham, UK

\subsection{6/gutjnl-2021-BSG.252}

Introduction There are clear guidelines regarding the diagnosis and management of Barrett's oesophagus, including diagnosis, screening, surveillance and management (1).

Previous studies have demonstrated that there is a limited role for endoscopic surveillance following oesophagectomy (2).

Currently there is no consensus on whether surveillance for Barrett's should be performed in such patients, or how to manage residual Barrett's post-operatively.

We audited our practice in the management of such patients at a tertiary referral centre.

Methods The records of patients whom underwent oesophagectomy for malignancy between January 1st 2010 and December 31st 2020 identified from EPR were reviewed.

Data obtained included demographics, date of surgery, histological tumour type, TNM staging and whether further endoscopies were performed in each case. Follow up endoscopy reports were assessed for evidence of residual Barrett's, dysplasia or neoplasia and its management.

Results 175 patients met the criteria for inclusion.

The mean age was 64 (22-82; median 65). The majority were male (76.6\%). Most cancers were adenocarcinoma (77\%).

Following surgery 66 patients had one or more OGD performed, the majority of whom had just one (41).

Two patients were identified as having Barrett's oesophagus at their follow up endoscopy, both of whom were placed on a surveillance program.

In those patients who had repeat endoscopy 3/66 had a cancer recurrence at OGD. However, these had already been identified prior to OGD via CT scan or found at surgery.

Only 6 patients had residual Barrett's oesophagus based on the upper margin of the resection specimen. One also had residual dysplasia at the resection margin. Of these patients only one had an OGD following their surgery, for surgical indications and none had any surveillance endoscopy. One patient with residual Barrett's was subsequently diagnosed with a recurrence of malignancy, initially picked up through CT.

Conclusions Very few patients who had an oesophagectomy had further endoscopy. No patients were diagnosed with recurrence at endoscopy. We are evolving our practice to eradication of Barrett's and dysplasia post-oesophagectomy, in line with the usual management of Barrett's with dysplasia. Further investigation is required to determine whether follow up endoscopy and treatment helps to mitigate the risk of cancer recurrence.

\section{REFERENCES}

1. Fitzgerald RC, di Pietro M, Ragunath $K$, et al. British Society of Gastroenterology guidelines on the diagnosis and management of Barrett's oesophagus. Gut. 2014:63(1):7.

2. Lou F, Sima CS, Adusumilli PS, et al. Esophageal cancer recurrence patterns and implications for surveillance. J Thorac Oncol. 2013;8(12):1558-62. 